\title{
Joint measurement of multiple noncommuting parameters
}

\author{
Jiamin Li, ${ }^{1}$ Yuhong Liu, ${ }^{1}$ Liang Cui, ${ }^{1}$ Nan Huo, ${ }^{1}$ Syed M. Assad, ${ }^{2}$ Xiaoying Li,${ }^{1, *}$ and Z. Y. Ou ${ }^{1,3, \dagger}$ \\ ${ }^{1}$ College of Precision Instrument and Opto-Electronics Engineering, Key Laboratory of Opto-Electronics Information Technology, \\ Ministry of Education, Tianjin University, Tianjin 300072, People's Republic of China \\ ${ }^{2}$ Department of Quantum Science, Australian National University, Canberra ACT 0200, Australia \\ ${ }^{3}$ Department of Physics, Indiana University-Purdue University Indianapolis, Indianapolis, Indiana 46202, USA
}

(Received 30 November 2017; published 29 May 2018)

\begin{abstract}
Although quantum metrology allows us to make precision measurements beyond the standard quantum limit, it mostly works on the measurement of only one observable due to the Heisenberg uncertainty relation on the measurement precision of noncommuting observables for one system. In this paper, we study the schemes of joint measurement of multiple observables which do not commute with each other using the quantum entanglement between two systems. We focus on analyzing the performance of a SU $(1,1)$ nonlinear interferometer on fulfilling the task of joint measurement. The results show that the information encoded in multiple noncommuting observables on an optical field can be simultaneously measured with a signal-to-noise ratio higher than the standard quantum limit, and the ultimate limit of each observable is still the Heisenberg limit. Moreover, we find a resource conservation rule for the joint measurement.
\end{abstract}

DOI: 10.1103/PhysRevA.97.052127

\section{INTRODUCTION}

Quantum metrology, which uses quantum resources to improve sensitivity beyond the classical limit in the estimation of relevant physical parameters, has been one of the frontier topics in the applications of quantum technology [1,2]. Most previous studies are committed to improving the signal-tonoise ratio (SNR) of a single parameter, such as precision measurement of a phase shift, and the basic idea is to reduce the quantum noise in the measurement with novel quantum states $[3,4]$. So far, the squeezed state has been widely applied in quantum precision measurement of a single parameter, such as gravitational wave detection [5]. In some applications, however, the information is embedded in two or more noncommuting observables. For example, information about the real and imaginary parts of the linear susceptibility of an optical medium is embedded in the phase and amplitude of a probe optical field passing through the medium in the form of small modulated signals. According to the Heisenberg uncertainty principle for two noncommuting observables, quantum noise reduction in one observable is inevitably accompanied by noise increase in the other. Therefore, the strategy of quantum noise reduction fails in measuring two noncommuting observables with sensitivity simultaneously higher than classical limit.

On the other hand, Heisenberg uncertainty involves in two conjugate quantities of one system, but the situation for two systems is completely different. Quantum entanglement allows perfect quantum correlations between two systems. Einstein, Podolsky, and Rosen (EPR) showed in a seminal paper [6] that there exists such a state of two-particle systems that exhibits perfect correlations not only between the positions of two

\footnotetext{
*xiaoyingli@tju.edu.cn

†zheyuou@tju.edu.cn
}

remotely located particles but also between their momenta. This is so because the difference of their position operators $\hat{x}_{1}-\hat{x}_{2}$ and the sum of their momenta operators $\hat{p}_{1}+\hat{p}_{2}$ commute: $\left[\hat{x}_{1}-\hat{x}_{2}, \hat{p}_{1}+\hat{p}_{2}\right]=0$. Such perfect correlations led to the famous EPR paradox about the incompleteness of quantum mechanics via a locality argument. The experimental realization of the EPR entangled state and the demonstration of the EPR paradox were first done in an optical system of nondegenerate parametric amplifier $[7,8]$ in which the two particles are the virtual harmonic oscillators representing two spatially separated modes of optical beams with $\hat{x}_{1,2} \propto \hat{a}_{1,2}^{\dagger}+$ $\hat{a}_{1,2} \equiv \hat{X}_{1,2}$ and $\hat{p}_{1,2} \propto i\left(\hat{a}_{1,2}^{\dagger}-\hat{a}_{1,2}\right) \equiv \hat{Y}_{1,2}$, where $\hat{a}_{i}^{\dagger}$ and $\hat{a}_{i}$ $(i=1,2)$ are the creation and annihilation operators of the two optical fields, respectively. These magic quantum nonlocal correlations of orthogonal observables give rise to quantum noise reduction by noise cancellation via $\hat{X}_{1}-\hat{X}_{2}$ and $\hat{Y}_{1}+\hat{Y}_{2}$ and can be employed for the simultaneous measurement of the phase and amplitude encoded in $\hat{Y}_{1}$ and $\hat{X}_{1}$ of one optical beam. This idea was first proposed in the form of quantum dense coding $[9,10]$ and was demonstrated experimentally in the joint measurement of two orthogonal observables with a precision beating standard quantum limit (SQL) [11,12].

Along a similar line of argument, quantum entanglement has also been used in quantum noise cancellation in an amplifier for noiseless quantum amplification [13,14]. This is exactly the underlying principle for the so-called $\operatorname{SU}(1,1)$ interferometer (SUI), a type of nonlinear interferometer that is based on nonlinear parametric processes for wave splitting and superposition. Proposed as early as 1986 by Yurke et al. [15], the SUI can in principle reach the Heisenberg limit in the precision measurement of a phase shift [16,17]. Although practical imperfections limited its ability to reach the ultimate precision, it was demonstrated that SUIs can still beat the standard quantum limit of phase measurement and are superior to traditional interferometers in a number of ways [17-21]. 
So far, for the SUI used for quantum enhanced phase measurement, only one of the two output ports is exploited. However, it turns out that the other SUI output port also contains information for the sensing field inside the interferometer [22], which can be used for amplitude measurement. In this paper, we will study the performance of the SUI in the application of simultaneously measuring noncommuting observables with precision beating SQL. We will find the optimum operation condition for achieving the highest SNR in the simultaneous measurement of each observable. Also, we will compare this scheme of joint measurement with others using classical light. Moreover, we will compare the performance of SUI respectively with one-beam and dual-beam function as the sensing field, which leads to a resource conservation rule for joint measurement. Furthermore, we will demonstrate that the ultimate limit of the precision in the joint measurement is still the Heisenberg limit.

The rest of the paper is organized as follows. We first briefly review the SNR of multiparameter measurement obtained using three typical classical schemes in Sec. II. Next, in Sec. III we study the quantum enhanced measurement schemes using quantum entanglement, including a quantum dense coding scheme and a SUI. In this section, we focus on analyzing the SUI as a platform for joint measurement and demonstrating its advantages over the dense coding scheme. In Sec. IV we discuss how the Heisenberg limit can be approached in both phase and amplitude measurement. Finally, we briefly conclude in Sec. V.

\section{JOINT MEASUREMENT SCHEMES WITH CLASSICAL LIGHT}

Before introducing the quantum enhanced joint measurement schemes, we start by first considering the measurement schemes using classical light sources. These give rise to SQL for the joint measurement of the information embedded in two noncommuting quadrature-phase amplitudes of an optical beam.

\section{A. Direct measurement}

To obtain the information on phase or amplitude carried by a probe beam, the simplest scheme is direct measurement realized using homodyne detection, as shown in Fig. 1. We assume the probe field $\hat{a}_{\text {in }}$ is in a coherent state $|\alpha\rangle$ with $|\alpha\rangle=|\alpha| e^{j \varphi_{0}}$ where $\alpha$ is a complex number and $\varphi_{0}$ is the initial phase. By passing the probe through an amplitude modulator (AM) and a phase modulator (PM), a weak phase modulation of $\delta \ll 1$ and a weak amplitude modulation $\epsilon \ll 1$

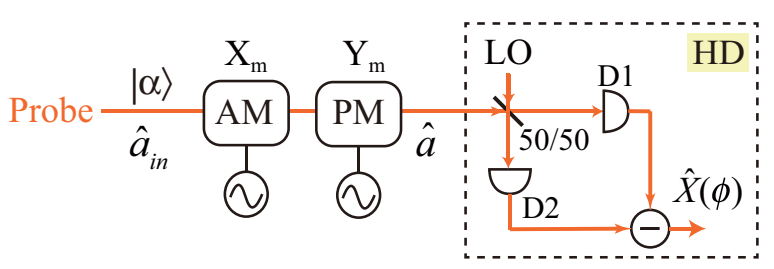

FIG. 1. Direct measurement scheme. AM, amplitude modulator; PM, phase modulator; HD, homodyne detection; D1 and D2, detectors; LO, local oscillator. simultaneously applied to the probe beam can be expressed as $e^{j \delta} \approx 1+j \delta$ and $e^{-\epsilon} \approx 1-\epsilon$, respectively. The modulated probe field is then expressed as $\hat{a}=\hat{a}_{\text {in }} e^{j \delta} e^{-\epsilon} \approx \hat{a}_{\text {in }}(1+j \delta-$ $\epsilon)$. On the other hand, a modulation signal of an arbitrary quadrature amplitude $X_{m}(\theta)=X_{m} \cos \theta+Y_{m} \sin \theta$ can be viewed as a combination of amplitude and phase modulations, where $X_{m}=X_{m}(0) \equiv \epsilon$ and $Y_{m}=X_{m}(\pi / 2) \equiv \delta$. Here, for consistency with the analysis hereinafter, $X_{m}$ and $Y_{m}$ are used to denote, respectively, the amplitude modulation and phase modulation, while $X_{m}(\theta)$ is used to specify a modulation signal of quadrature amplitude at arbitrary angle $\theta$. The modulated signal of the probe beam can be directly measured using homodyne detection (HD), which consists of a 50/50 beam splitter (BS) and two detectors (D1 and D2). When the phase of the local oscillator (LO) is set to $\phi$, the difference between the photocurrents of D1 and D2 gives the measurement of the modulated probe beam $\hat{X}(\phi)=\hat{a} e^{-j \phi}+\hat{a}^{\dagger} e^{j \phi}$.

Defining $\hat{X} \equiv \hat{X}\left(\varphi_{0}\right)$ and $\hat{Y} \equiv \hat{X}\left(\varphi_{0}+\pi / 2\right)$ as two conjugate observables, it is straightforward to deduce that the measurement of a probe beam for $\hat{X}$ gives the amplitude modulation signal $\langle\hat{X}\rangle=2|\alpha| \epsilon=2|\alpha| X_{m}$, whereas that for $\hat{Y}$ gives the phase modulation $\langle\hat{Y}\rangle=2|\alpha| \delta=2|\alpha| Y_{m}$. In general, the measurement of $\hat{X}\left(\varphi_{0}+\theta\right)$ measures the modulated quadrature amplitude at arbitrary angle $\left\langle\hat{X}\left(\varphi_{0}+\theta\right)\right\rangle=2|\alpha| X_{m}(\theta)$. On the other hand, for the probe in a coherent state, its noise is independent of the phase of LO, i.e., $\left\langle\Delta^{2} \hat{X}(\phi)\right\rangle=1$. So we have the SNR for the direct measurement of phase or amplitude:

$$
\begin{aligned}
& S N R_{D M}(\hat{Y})=\frac{\langle\hat{Y}\rangle^{2}}{\left\langle\Delta \hat{Y}^{2}\right\rangle}=4 I_{\mathrm{ps}} \delta^{2} \text { or } \\
& S N R_{D M}(\hat{X})=\frac{\langle\hat{X}\rangle^{2}}{\left\langle\Delta \hat{X}^{2}\right\rangle}=4 I_{\mathrm{ps}} \epsilon^{2},
\end{aligned}
$$

where $I_{\mathrm{ps}} \equiv|\alpha|^{2}$ is the intensity or photon number for the probe-sensing beam, and the subscript "DM" represents the direct measurement scheme in Fig. 1.

\section{B. Beam-splitting method}

However, the scheme in Fig. 1 can make direct measurement of only one observable at one time. In the other words, either phase or amplitude can be measured at one time. A straightforward method of jointly measuring $\hat{X}$ and $\hat{Y}$ is to split the modulated probe beam into two with a beam splitter (BS), as shown in Fig. 2. The two quadrature-phase amplitudes $\hat{X}$ and $\hat{Y}$ can be simultaneously measured at the two outputs of BS by using HD1 and HD2, respectively. Since the probe

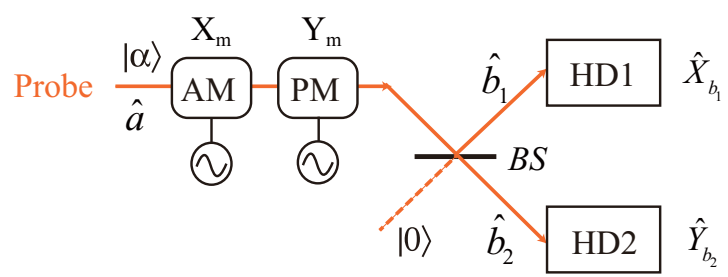

FIG. 2. Joint measurement scheme using a beam splitter (BS). AM, amplitude modulator; PM, phase modulator; HD, homodyne detection. 
is in an ideal coherent state $|\alpha\rangle$, its noise is simply given by $\left\langle\Delta^{2} \hat{X}(\phi)\right\rangle=1$ (the same as the vacuum noise). If the detection efficiency of each HD device is perfect, and the relative phase of each HD is properly locked, the SNRs of the simultaneously measured phase and amplitude signals are expressed as

$$
S N R_{B S}\left(\hat{Y}_{b_{2}}\right)=4 T I_{\mathrm{ps}} \delta^{2}, \quad S N R_{B S}\left(\hat{X}_{b_{1}}\right)=4 R I_{\mathrm{ps}} \epsilon^{2}
$$

where the subscripts " $b_{1}$ " and " $b_{2}$ " denote the fields at two BS ports, and $T$ and $R$ with $T+R=1$ the transmissivity and reflectivity of the BS, respectively. When the sizes of phase modulation and amplitude modulation are equal, i.e., $\delta=\epsilon$, we have

$$
S N R_{B S}\left(\hat{Y}_{b_{2}}\right)+S N R_{B S}\left(\hat{X}_{b_{1}}\right)=S N R_{D M}(\hat{Y})=S N R_{D M}(\hat{X})
$$

From Eq. (3), we find the total sum of the SNRs for joint measurement of phase and amplitude is equal to the SNR of phase or amplitude obtained by direct measurement in Fig. 1, in which all the resources are consumed on one observable. In other word, the joint measurement can be viewed as the partition of the total resource into two observables.

For the BS with the splitting ratio $T=R=1 / 2$, we have

$$
S N R_{B S}\left(\hat{Y}_{b_{2}}\right)=2 I_{\mathrm{ps}} \delta^{2}, \quad S N R_{B S}\left(\hat{X}_{b_{1}}\right)=2 I_{\mathrm{ps}} \epsilon^{2} .
$$

Notice that $\quad S N R_{B S}\left(\hat{Y}_{b_{2}}\right)=S N R_{D M}(\hat{Y}) / 2 \quad$ and $S N R_{B S}\left(\hat{X}_{b_{1}}\right)=S N R_{D M}(\hat{X}) / 2$ are referred to as the SQL of the joint measurement. Comparing with the SNR obtained by the direct measurement method [see Eq. (1)], there is a $3 \mathrm{~dB}$ reduction for the SNR measured at each output port in Fig. 2, which is originated from the vacuum $|0\rangle$ entering from the unused BS port.

\section{Beam-splitting scheme with an optical parametric amplifier}

In a real experiment, the measured SNRs are usually smaller than those given by Eq. (4) due to the nonideal HD detection efficienciess. One way to mitigate the influence of detection loss is to replace the 50/50 BS in Fig. 2 with a conventional optical parametric amplifier (OPA), as shown in Fig. 3. As we will show, the OPA scheme at high gain is similar to the BS scheme in Fig. 2. So SNRs of joint measurement performed using an OPA can be viewed as a direct comparison with the quantum scheme of an SUI (see Sec. III B for details).

An optical parametric amplifier has its input probe beam amplified at the signal port and in the meantime also outputs another field called the idler, which contains the information for the input probe field as well [23]. From the input-output

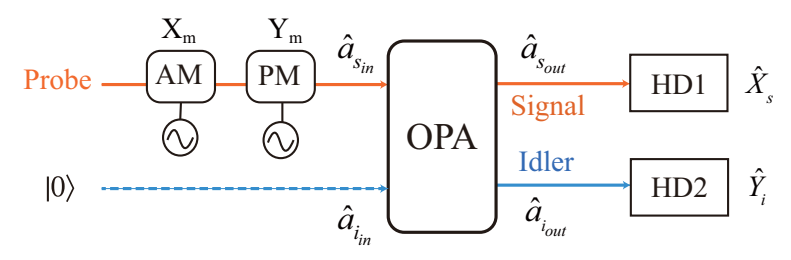

FIG. 3. Joint measurement scheme using an optical parametric amplifier (OPA) as a beam splitter. AM, amplitude modulator; PM, phase modulator; $\mathrm{HD}$, homodyne detection. relation of the OPA:

$$
\hat{a}_{s_{\text {out }}}=G \hat{a}_{s_{\text {in }}}+g \hat{a}_{i_{\text {in }}}^{\dagger}, \quad \hat{a}_{i_{\text {out }}}=G \hat{a}_{i_{\text {in }}}+g \hat{a}_{s_{\text {in }}}^{\dagger}
$$

with $G^{2}-g^{2}=1$, where $G, g$ are the amplitude gains of OPA, the signal probe input $\hat{a}_{s_{\mathrm{in}}}$ is in a coherent state, and idler input $\hat{a}_{i_{\text {in }}}$ is in vacuum. The signal and idler output fields have the averages of $\left\langle\hat{a}_{s_{\text {out }}}\right\rangle=G \alpha$ and $\left\langle\hat{a}_{i_{\text {out }}}\right\rangle=g \alpha^{*}$. Thus, an OPA can act as a BS through which the information on phase and amplitude modulation encoded on a probe beam is distributed to the signal and idler output ports.

From Eq. (5), it is straightforward to deduce the average powers of the phase and amplitude modulations measured by HD1 and HD2,

$$
\left\langle\hat{X}_{s_{\text {out }}}\right\rangle^{2}=4 G^{2} I_{\mathrm{ps}} \epsilon^{2}, \quad\left\langle\hat{Y}_{i_{\text {out }}}\right\rangle^{2}=4 g^{2} I_{\mathrm{ps}} \delta^{2},
$$

where the subscripts $s_{\text {out }}$ and $i_{\text {out }}$, respectively, indicate the signal and idler outputs. Moreover, according to the noise measured at the signal and idler outputs,

$$
\left\langle\Delta^{2} \hat{X}_{s_{\text {out }}}\right\rangle=\left\langle\Delta^{2} \hat{Y}_{i_{\text {out }}}\right\rangle=G^{2}+g^{2},
$$

we arrive at the SNRs of phase and amplitude:

$$
S N R_{\mathrm{Amp}}\left(\hat{X}_{s}\right)=\frac{4 G^{2} I_{\mathrm{ps}} \epsilon^{2}}{G^{2}+g^{2}}, \quad S N R_{\mathrm{Amp}}\left(\hat{Y}_{i}\right)=\frac{4 g^{2} I_{\mathrm{ps}} \delta^{2}}{G^{2}+g^{2}},
$$

where the subscript "Amp" denotes that the results are for the conventional amplifier scheme in Fig. 3. In the case of $G \rightarrow \infty$, SNRs in Eq. (8) are rewritten as

$$
\begin{gathered}
S N R_{\mathrm{Amp}}\left(\hat{X}_{s}\right)=\frac{4 G^{2} I_{\mathrm{ps}} \epsilon^{2}}{G^{2}+g^{2}} \rightarrow 2 I_{\mathrm{ps}} \epsilon^{2}, \\
S N R_{\mathrm{Amp}}\left(\hat{Y}_{i}\right)=\frac{4 g^{2} I_{\mathrm{ps}} \delta^{2}}{G^{2}+g^{2}} \rightarrow 2 I_{\mathrm{ps}} \delta^{2} .
\end{gathered}
$$

It is obvious that the SNRs in Eq. (9) are the same as the SQL in Eq. (4). Notice that for the case of $\delta=\epsilon$, similar to Eq. (3), we again have the resource partition relation:

$$
S N R_{\mathrm{Amp}}\left(\hat{X}_{s}\right)+S N R_{\mathrm{Amp}}\left(\hat{Y}_{i}\right)=S N R_{D M}(\hat{X}) .
$$

To illustrate the loss tolerance advantage of OPA scheme, let's analyze the influence of detection efficiency on the SNRs of joint measurement. In general, the detection loss $L_{d}$ is modeled by placing a BS in front of each HD. The BS transmissivity is viewed as $1-L_{d}$, and vacuum field $\hat{v}$ is coupled into the detected field through the nonideal transmissivity. Taking the OPA signal output as an example, the operator of the detected field is given by $\hat{a}_{s_{\text {out }}}^{\prime}=\sqrt{\left(1-L_{d}\right)} \hat{a}_{s_{\text {out }}}+\sqrt{L_{d}} \hat{v}$. So the measured average power of amplitude,

$$
\left\langle\hat{X}_{s_{\text {out }}}^{\prime}\right\rangle^{2}=\left(1-L_{d}\right)\left\langle\hat{X}_{s_{\text {out }}}\right\rangle^{2}
$$

decreases with the increase of detection loss. Meanwhile, the measured noise

$$
\left\langle\Delta^{2} \hat{X}_{s_{\text {out }}^{\prime}}\right\rangle=\left(1-L_{d}\right)\left\langle\Delta^{2} \hat{X}_{s_{\text {out }}}\right\rangle+L_{d}
$$

accordingly changes because of the vacuum noise coupled in through loss. Since the OPA noise [see Eq. (7)] is much larger than that of vacuum, particularly in the high-gain regime, the noise in Eq. (12) decreases with the increase of $L_{d}$, and the decrease rate is about the same as that of the signal power 


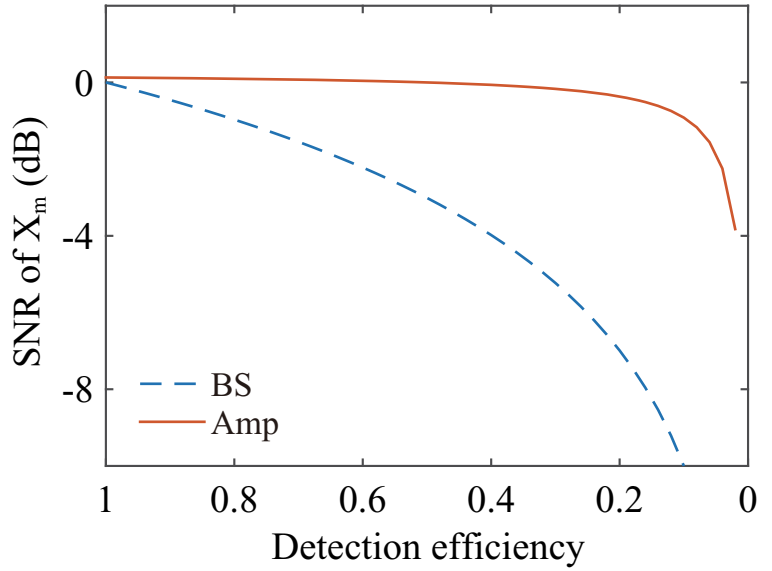

FIG. 4. SNR of the measured amplitude $X_{m}$ as a function of detection efficiency $1-L_{d}$. Solid curve and dashed curve, respectively, represent the joint measurement results for the schemes in Figs. 3 and 2 . In the calculation, $I_{\mathrm{ps}} \epsilon^{2}=1 / 2$, and the OPA amplitude gain is $g=5$.

in Eq. (11). Therefore, the SNR of the joint measurement scheme in Fig. 3 is not sensitive to detection loss when the OPA amplitude gain and the HD detection efficiency are not too low, as shown by the solid curve in Fig. 4. As a comparison, we also plot the SNR of amplitude modulation measured by the BS scheme (Fig. 2) as a function of the detection efficiency, as shown by the dashed curve in Fig. 4. Clearly, the SNRs for the BS scheme significantly decrease with the detection efficiency. This is because the noise at each output of the BS scheme is always at the vacuum noise level, while the measured average power of signal modulation is proportional to the detection efficiency.

\section{JOINT MEASUREMENT SCHEMES WITH QUANTUM FIELDS}

In this section, we will analyze the quantum enhanced joint measurement by briefly reviewing the quantum dense coding scheme for quantum dense metrology at first. Then we will focus on analyzing the SUI in the application of joint measurement. In addition to studying its optimum operation condition for achieving the maximized SNRs in joint measurement, we will compare the SUI performance with one-beam and dual-beam function as the sensing field, respectively. Finally, we will discuss the resource conservation rule when two noncommuting observables are simultaneously measured.

\section{A. Quantum dense coding scheme for quantum dense metrology}

To implement the quantum enhanced joint measurement, we need to design a simultaneous measurement on both $\hat{X}_{1}-\hat{X}_{2}$ and $\hat{Y}_{1}+\hat{Y}_{2}$ of a pair of entangled fields to extract the information carried by a probe. This is the quantum dense coding scheme [9] shown in Fig. 5. The two EPR entangled fields labeled $\hat{a}_{s_{\text {out }}}, \hat{a}_{i_{\text {out }}}$ are generated from an OPA $[7,8,11]$. One of the entangled fields is encoded with both phase and amplitude information by a phase modulator (PM) and an amplitude modulator (AM), respectively. When the modulated field and the other half of entangled field, now labeled $\hat{a}_{1}, \hat{a}_{2}$,

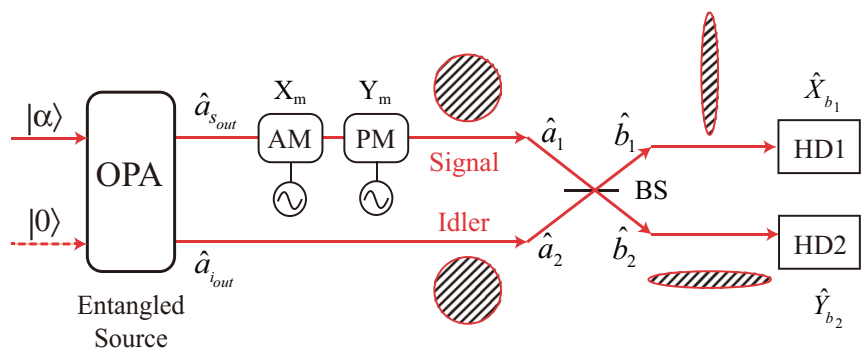

FIG. 5. Dense coding scheme for quantum dense metrology. OPA, optical parametric amplifier; AM, amplitude modulator; PM, phase modulator; HD, homodyne detection. The shadows illustrate the noise distribution of the fields at the two BS inputs and outputs.

are superimposed at a 50/50 BS, the two BS outputs are given by

$$
\hat{b}_{1}=\left(\hat{a}_{1}-\hat{a}_{2}\right) / \sqrt{2}, \quad \hat{b}_{2}=\left(\hat{a}_{2}+\hat{a}_{1}\right) / \sqrt{2} .
$$

If we measure $\hat{X}_{b_{1}}=\left(\hat{X}_{a_{1}}-\hat{X}_{a_{2}}\right) / \sqrt{2}$ at the $\hat{b}_{1}$ port and $\hat{Y}_{b_{2}}=\left(\hat{Y}_{a_{1}}+\hat{Y}_{a_{2}}\right) / \sqrt{2}$ at the $\hat{b}_{2}$ port using HD1 and HD2, respectively, we can achieve the simultaneous measurement of $\hat{X}_{a_{1}}-\hat{X}_{a_{2}}$ and $\hat{Y}_{a_{1}}+\hat{Y}_{a_{2}}$ [8-11]. So the amplitude and phase modulation signals carried by $\hat{a}_{1}$ can be obtained by simultaneously measuring $\hat{X}_{b_{1}}$ and $\hat{Y}_{b_{2}}$, whose noise fluctuations are lower than shot noise level (SNL), and the measurement sensitivities are beyond SQL.

In Fig. 5 the two OPA outputs are described by

$$
\hat{a}_{s_{\mathrm{out}}}=G \hat{a}_{s_{\mathrm{in}}}+g \hat{a}_{i_{\mathrm{in}}}^{\dagger}, \quad \hat{a}_{i_{\mathrm{out}}}=G \hat{a}_{i_{\mathrm{in}}}+g \hat{a}_{s_{\mathrm{in}}}^{\dagger},
$$

where $\hat{a}_{s_{\text {in }}}$ in coherent state $|\alpha\rangle$ is the weak signal input, and $\hat{a}_{i_{\text {in }}}$ in vacuum state $|0\rangle$ is the idler input. The modulated signal and its correlated field before the BS, expressed as $\hat{a}_{1}=$ $\hat{a}_{S_{\text {out }}}(1+j \delta-\epsilon)$ and $\hat{a}_{2}=\hat{a}_{i_{\text {out }}}$, are then combined by the BS. At the two BS outputs, the operators $\hat{X}_{b_{1}}=\hat{b}_{1} e^{-j \varphi_{0}}+\hat{b}_{1}^{\dagger} e^{j \varphi_{0}}$ and $\hat{Y}_{b_{2}}=\left(\hat{b}_{2} e^{-j \varphi_{0}}-\hat{b}_{2}^{\dagger} e^{j \varphi_{0}}\right) / j\left(e^{j \varphi_{0}} \equiv \alpha /|\alpha|\right)$ are measured by HD1 and HD2 with $\phi_{1}=0$ and $\phi_{2}=\pi / 2$, respectively. The measurement gives the powers of modulated amplitude and phase signals:

$$
\left\langle\hat{X}_{b_{1}}\right\rangle^{2}=2 I_{\mathrm{ps}} \epsilon^{2}, \quad\left\langle\hat{Y}_{b_{2}}\right\rangle^{2}=2 I_{\mathrm{ps}} \delta^{2},
$$

where $I_{\mathrm{ps}}=\left\langle\hat{a}_{s_{\text {out }}}^{\dagger} \hat{a}_{s_{\text {out }}}\right\rangle=G_{1}^{2}|\alpha|^{2}\left(|\alpha|^{2} \gg 1\right)$, with $|\alpha|^{2}$ denoting the intensity of the seed injection, is the intensity of the probe beam. Meanwhile, the noise fluctuations measured at the two BS outputs are given by

$$
\begin{gathered}
\left\langle\Delta^{2} \hat{X}_{b_{1}}\right\rangle=\left\langle\Delta^{2}\left(\hat{X}_{a_{1}}-\hat{X}_{a_{2}}\right)\right\rangle=1 /(G+g)^{2}, \\
\left\langle\Delta^{2} \hat{Y}_{b_{2}}\right\rangle=\left\langle\Delta^{2}\left(\hat{Y}_{a_{1}}+\hat{Y}_{a_{2}}\right)\right\rangle=1 /(G+g)^{2},
\end{gathered}
$$

which are lower than the SNL due to the entanglement correlation between the fields $\hat{a}_{1}$ and $\hat{a}_{2}$. Accordingly, the SNRs of the jointly measured amplitude and phase modulations are

$$
\begin{aligned}
& S N R_{D C}\left(\hat{X}_{b_{1}}\right)=2(G+g)^{2} I_{\mathrm{ps}} \epsilon^{2}, \\
& S N R_{D C}\left(\hat{Y}_{b_{2}}\right)=2(G+g)^{2} I_{\mathrm{ps}} \delta^{2},
\end{aligned}
$$

where the subscript "DC" is used to denote the dense coding scheme. Comparing with the SQLs in Eq. (4), it is obvious that the SNRs of joint measurement are improved using the 


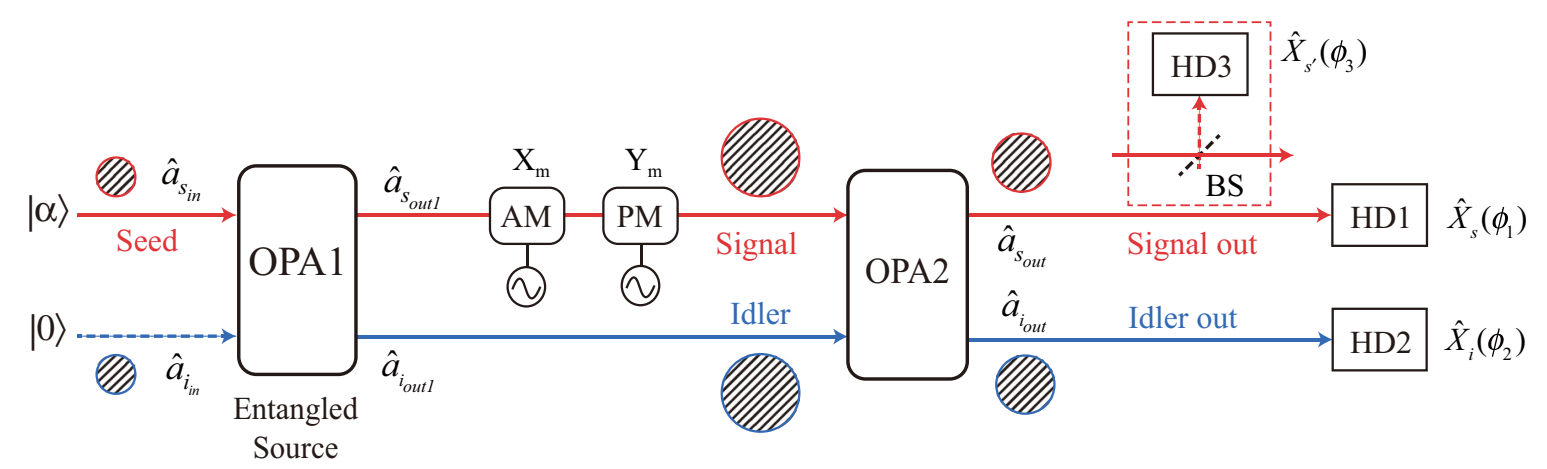

FIG. 6. SUI formed by OPA1 and OPA2 for the joint measurement of multiple noncommuting quadratures. AM, amplitude modulator; PM, phase modulator; OPA, optical parametric amplifier; HD, homodyne detection; BS, beam splitter. The shadows illustrate the noise distribution at different ports of OPAs.

EPR entanglement with a factor of $(G+g)^{2}$. Moreover, it is worth noting that for the dense coding scheme, it is critical to carefully set the LOs of HD1 and HD2 to achieve the best quantum enhancement, because noise at each BS output highly depends on the phase of LOs, $\phi_{1}$ and $\phi_{2}$, as shown by the shadows in Fig. 5.

\section{B. Nondegenerate SUI with one-beam sensing}

The BS in the dense coding scheme in Fig. 5 can realize coherent combination only when the frequencies of the two entangled fields are the same. To coherently combine two entangled fields with nondegenerate frequencies, we resort to an SUI, in which the BS in Fig. 5 is replaced with an optical parametric amplifier.

As shown in Fig. 6, the SUI consists of two OPAs, which respectively act as beam splitters for wave splitting and superposition. What makes it different from a conventional linear interferometer is that the two fields split by OPA 1 are now correlated in noise, which can be canceled out in the second OPA due to destructive quantum interference. When the modulation signals encoded in the signal beam out of OPA1 is amplified by OPA2, a noiseless amplification can be achieved at each SUI output [22].

In Fig. 6, the seed injection and vacuum, respectively, at two input ports of OPA 1 are denoted as the field operators $\hat{a}_{s_{\mathrm{in}}}$ and $\hat{a}_{i_{\text {in }}}$. The two entangled quantum fields out of OPA 1 are referred to as $\hat{a}_{s_{\text {out } 1}}$ and $\hat{a}_{i_{\text {out } 1}}$, respectively. The signal probe field, encoded with the information for multiple noncommuting observables by successively passing through an AM and a PM, is sent into the OPA2 together the idler field. The information carried by the probe beam is then amplified by OPA2, whose outputs are denoted as $\hat{a}_{S_{\text {out }}}$ and $\hat{a}_{i_{\text {out }}}$, respectively.

The theoretical analyses in Refs. [22,24] show that OPA2 of the SUI functions as a phase-insensitive amplifier for the information carried by the signal probe beam. When the probe is embedded with the weakly modulated phase and amplitude signals, $\delta \ll 1$ and $\epsilon \ll 1$, the average powers of the two observables measured, respectively, at the signal and idler output ports by HD1 and HD2 are given by

$$
\left\langle\hat{X}_{s_{\text {out }}}\right\rangle^{2}=4 G_{2}^{2} I_{\mathrm{ps}} \epsilon^{2}, \quad\left\langle\hat{Y}_{i_{\text {out }}}\right\rangle^{2}=4 g_{2}^{2} I_{\mathrm{ps}} \delta^{2},
$$

where $I_{\mathrm{ps}}=\left\langle\hat{a}_{s_{\text {out } 1}}^{\dagger} \hat{a}_{s_{\text {out } 1}}\right\rangle=G_{1}^{2}|\alpha|^{2}\left(|\alpha|^{2} \gg 1\right)$. However, the noise fluctuation at each SUI output port is sensitive to the relative phase between the pump and the two input fields of OPA2. For brevity, the relative phase is represented by introducing a phase shift $\varphi$ to the idler field $\hat{a}_{i_{\text {out }}}$. When OPA 2 is operated at the deamplification condition, i.e., $\varphi=\pi[22,24]$, the intensities at the two SUI outputs are minimum. In this case, the noise fluctuation measured at each output port takes the minimum and is expressed as

$$
\begin{aligned}
\left\langle\Delta^{2} \hat{X}_{s_{\text {out }}}\left(\phi_{1}\right)\right\rangle & =\left(G_{2} G_{1}-g_{1} g_{2}\right)^{2}+\left(G_{1} g_{2}-G_{2} g_{1}\right)^{2} \\
& =\left\langle\Delta^{2} \hat{X}_{i_{\text {out }}}\left(\phi_{2}\right)\right\rangle,
\end{aligned}
$$

where $g_{1}$ and $g_{2}$, satisfying the relation $G_{k}^{2}-g_{k}^{2}=1(k=1,2)$, are the amplitude gains of OPA1 and OPA2, respectively. Notice that the SUI output noise is independent of the quadrature phase angles $\phi_{1}, \phi_{2}$ of LOs in homodyne detections, as represented by the circularly shaped shadows in Fig. 6. This is a unique property for SUI.

In the case of $g_{1}=g_{2}$, the noise fluctuation takes the absolute minimum $\left\langle\Delta^{2} \hat{X}_{s_{\text {out }}}\left(\phi_{1}\right)\right\rangle=\left\langle\Delta^{2} \hat{X}_{i_{\text {out }}}\left(\phi_{2}\right)\right\rangle=1$, which means that the SUI output noise level is the same as the vacuum state or coherent state even after the amplification of two OPAs. Comparing with the conventional OPA scheme [see Eqs. (7) and (8)], one sees that although the modulation signals of phase and amplitude, carried by the probe beam, experience the same gain of $g=g_{2}=g_{1}$ in both cases, the SUI noise is reduced by a factor of $1 /\left(G_{1}^{2}+g_{1}^{2}\right)$ because of a destructive quantum interference effect for noise cancellation [14].

According to Eqs. (18) and (19), we have the SNRs of phase and amplitude simultaneously measured at the SUI signal and idler outputs as

$$
\begin{aligned}
& \operatorname{SNR}_{S U I}\left(\hat{X}_{s}\right)=\frac{4 G_{2}^{2} I_{\mathrm{ps}} \epsilon^{2}}{\left(G_{2} G_{1}-g_{1} g_{2}\right)^{2}+\left(G_{1} g_{2}-G_{2} g_{1}\right)^{2}}, \\
& \operatorname{SNR}_{S U I}\left(\hat{Y}_{i}\right)=\frac{4 g_{2}^{2} I_{\mathrm{ps}} \delta^{2}}{\left(G_{2} G_{1}-g_{1} g_{2}\right)^{2}+\left(G_{1} g_{2}-G_{2} g_{1}\right)^{2}},
\end{aligned}
$$

where the subscript "SUI" represents the quantum measurement scheme in Fig. 6. For OPA1 with a fixed gain $g_{1}$, the maximum SNRs in signal and idler outputs are obtained at $g_{2}=2 g_{1} G_{1}$ and $g_{2} \rightarrow \infty$, respectively. When the gain of OPA2 approaches infinity, i.e., $g_{2} \rightarrow \infty$, Eq. (20) is rewritten 

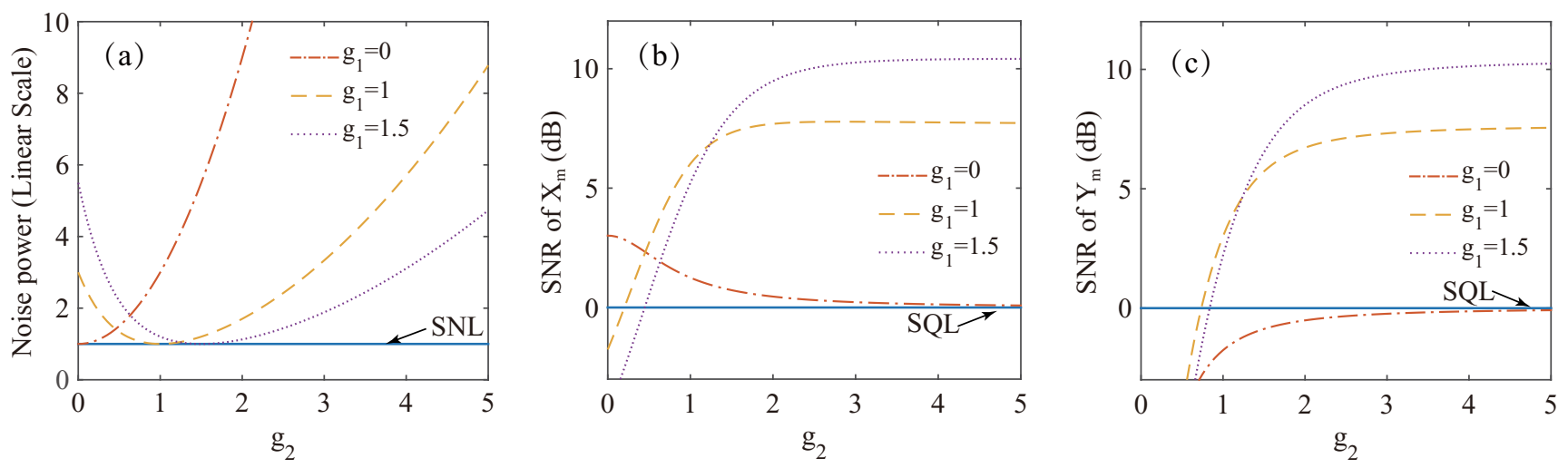

FIG. 7. Noise power and SNRs of joint measurement as a function of $g_{2}$ when gain of OPA1 is fixed at different levels. Panel (a) is the noise power at each SUI output port (b) and (c) are the SNRs of amplitude modulation signal $X_{m}=\epsilon$ and phase signal $Y_{m}=\delta$, respectively, measured at the SUI signal and idler outputs. In the calculation, $I_{\mathrm{ps}} \epsilon^{2}=1 / 2$; SNL, shot noise level; SQL, standard quantum limit.

as

$$
\begin{aligned}
S N R_{S U I}\left(\hat{X}_{s}\right) & =2\left(G_{1}+g_{1}\right)^{2} I_{\mathrm{ps}} \epsilon^{2}, \\
S N R_{S U I}\left(\hat{Y}_{i}\right) & =2\left(G_{1}+g_{1}\right)^{2} I_{\mathrm{ps}} \delta^{2} .
\end{aligned}
$$

Comparing Eq. (21) with SQL [see Eq. (9)], it is obvious that for the probe field with fixed intensity $I_{\mathrm{ps}}$, the SUI can achieve a better SNR than the classical OPA scheme with an enhancement factor of

$$
\frac{S N R_{S U I}\left(\hat{X}_{s}\right)}{S N R_{\mathrm{Amp}}\left(\hat{X}_{s}\right)}=\frac{S N R_{S U I}\left(\hat{Y}_{i}\right)}{S N R_{\mathrm{Amp}}\left(\hat{Y}_{i}\right)}=\left(G_{1}+g_{1}\right)^{2} .
$$

This improvement factor in Eq. (22) is originated from the noise cancellation due to the quantum correlations of the fields out of OPA1.

In addition to the joint measurement of two conjugate variables, such as phase and amplitude, SUI can simultaneously measure two quadrature amplitudes at arbitrary angles, i.e., $X_{m}(\theta)=X_{m} \cos \theta+Y_{m} \sin \theta$ at $\theta=\theta_{1}, \theta_{2}$, with SNRs surpassing the SQL when the LOs of HD1 and HD2 are properly adjusted. With the change of LO phase of $\phi_{i}(i=1,2)$, one may expect a different, likely higher, noise level. This is true for the quantum scheme in Fig. 5 but is not the case for SUI. According to Eq. (19), the noise at the SUI signal and idler output ports is irrelevant to the angle of quadrature amplitude. For the probe beam encoded with two quadrature-phase amplitudes $X_{m}\left(\theta_{1}\right)$ and $X_{m}\left(\theta_{2}\right)$, the homodyne measurement of $\hat{X}_{s}\left(\phi_{1}\right)$ and $\hat{X}_{i}\left(\phi_{2}\right)$ $\left(\phi_{1,2}=\theta_{1,2}\right)$ at the two outputs will simultaneously decode the information of $X_{m}\left(\theta_{1}\right)$ and $X_{m}\left(\theta_{2}\right)$ with SNRs expressed as

$$
\begin{aligned}
& S N R_{S U I}\left(\hat{X}_{s}\left(\phi_{1}\right)\right)=2\left(G_{1}+g_{1}\right)^{2} I_{\mathrm{ps}} X_{m}\left(\theta_{1}\right)^{2}, \\
& S N R_{S U I}\left(\hat{X}_{i}\left(\phi_{2}\right)\right)=2\left(G_{1}+g_{1}\right)^{2} I_{\mathrm{ps}} X_{m}\left(\theta_{2}\right)^{2} .
\end{aligned}
$$

Comparing with the SQL, it is clear that the improvement factor of SNRs in Eq. (23) is the same as in Eq. (22).

It is worth noting that for the SUI scheme, the optimum condition for achieving the absolute minimum noise at the two output ports is different from that for obtaining the maximum SNRs in joint measurement. For OPA 1 with a fixed gain $g_{1}$, the former is achieved for OPA2 with the same gain as OPA $1\left(g_{1}=\right.$ $g_{2}$ ), while the latter is obtained for OPA2 with gain approaching infinity $\left(g_{2} \rightarrow \infty\right)$. To better understand this difference, we calculate from Eqs. (19) and (20) the noise and SNRs measured at each SUI output when $g_{1}$ takes different values. The results in Fig. 7 are calculated by assuming $I_{\mathrm{ps}} \epsilon^{2}=1 / 2$. Since the noise powers at signal and idler outputs are same [see Eq. (19)], we calculated the noise at signal output and show the results in Fig. 7(a). Moreover, we plot the SNR as a function of $g_{2}$ to show the variation trend of simultaneously measured of amplitude and phase modulation, $X_{m}, Y_{m}$ in Figs. 7(b) and 7(c), respectively. For ease of comparison, the corresponding SNL $\left(\left\langle\Delta^{2} \hat{X}(\phi)\right\rangle=1\right)$ and SQL of SNRs obtained by substituting $g_{1}=0$ and $g_{2} \rightarrow \infty$ in Eqs. (19) and (20) are depicted in Fig. 7 as well. It is clear that minimum noise at SNL is achieved for $g_{2}=g_{1}$, at which the SNRs also beat SQL. However, the highest SNRs for a given $g_{1}$ are not obtained under the condition of $g_{1}=g_{2}$. For a fixed $g_{1}\left(g_{1} \neq 0\right)$, the SNR continues to increase with $g_{2}$ when $g_{2}>g_{1}$, and the optimum SNR is obtained under the condition of $g_{2} \gg g_{1}$. From Fig. 7, we find that the optimum SNR depends only on $g_{1}$ and is better than the SNR at $g_{1}=g_{2}$ by about $3 \mathrm{~dB}$ when $g_{1} \gg 1$.

Notice that the optimum improvement factor of SNR for SUI given in Eq. (22) is the same as that for the quantum dense coding scheme in Eq. (17). Why is the optimum SNR obtained at $g_{2} \rightarrow \infty$ ? We think this is because OPA2 functions as a $50 / 50$ BS when $g_{2} \rightarrow \infty$. Therefore, in the sense of coherently mixing the two correlated fields, the role of OPA2 operating in the high-gain regime is the same as the 50/50 BS in Fig. 5.

In the discussion above, we set the operating point of the SUI at the dark fringe by adjusting the overall phase of the interferometer to $\varphi=\pi$. This is because the maximum quantum noise reduction occurs at the dark fringe due to destructive quantum interference. Thus, $\varphi=\pi$ should be the optimum operating point for the SUI. Since we just showed that the SUI with $g_{2} \gg g_{1}$ is equivalent to the quantum dense coding scheme in Fig. 5, this optimum operating point can also be viewed as an equivalent of the optimum squeezing at the two outputs of the BS in Fig. 5.

The improvement factors of the quantum enhanced joint measurement for the two schemes in Figs. 5 and 6 are the same, but the SUI surpasses the dense coding scheme in three aspects. First, the SUI can utilize the EPR correlation between two fields with different wavelengths. Second, the SUI noise reduction does not depend on the LO phase in homodyne detection, 


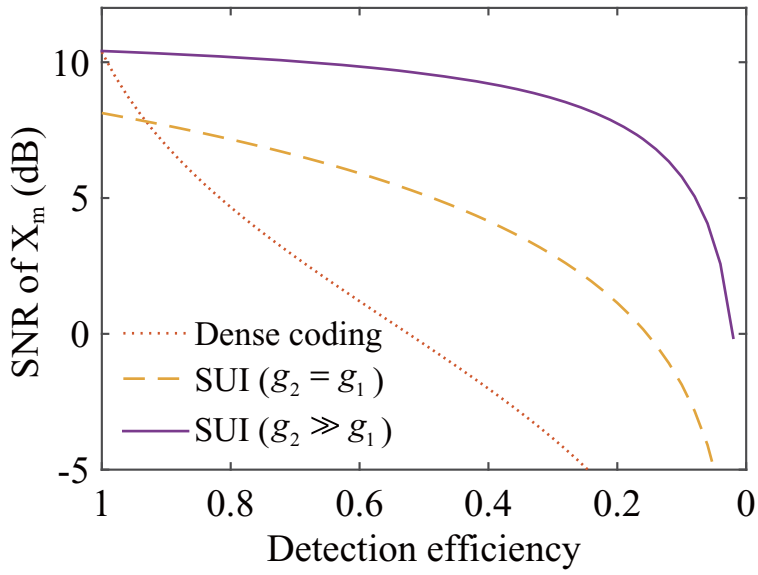

FIG. 8. The SNR of measured amplitude modulation of $X_{m}$ versus detection efficiency for both the SUI and dense coding scheme. In the calculation, $I_{\mathrm{ps}} \epsilon^{2}=1 / 2$; the SUI is working under the condition of $g_{1}=g_{2}=1.5$ (dashed curve) and $g_{2}=5 \gg g_{1}=1.5$ (solid curve), respectively; the gain of OPA in dense coding scheme is $g=1.5$; and the corresponding SQL of joint measurement is $0 \mathrm{~dB}$.

and the improvement in SNRs does not vary with the angles of the quadrature-phase amplitudes encoded on the probe. Third, the influence of the detection loss on SNR is diminished because the vacuum noise introduced through loss is negligible compared to the noise at the SUI outputs [see Fig. 7(a) and Eq. (12)] [19]. The first two points have been well presented in the analysis above. To illustrate the third advantage, we plot the SNRs as a function of detection efficiency when the gain of OPA2 in the SUI is set, respectively, to achieve optimum SNR ( $g_{2} \gg g_{1}$, solid curve) and lowest noise $\left(g_{1}=g_{2}\right.$, dashed curve) by taking the jointly measured amplitude as an example, as shown in Fig. 8. In the calculation, similar to the deduction of Eqs. (11) and (12), the nonideal detection is modeled as an insertion loss of the BS placed in front of HD. As a comparison, the relation between SNR and detection efficiency for the dense coding scheme is depicted in Fig. 8 as well. One sees that with the decrease of detection efficiency, the SNR value for the dense coding scheme quickly decreases (dotted curve), while the SNR downtrend for the SUI scheme is very slow, particularly for the SUI with $g_{2} \gg g_{1}$. Previous experimental demonstration of the loss-insensitive property was performed by the SUI under the operation condition of $g_{2}=g_{1}[18,25]$. Our results in Fig. 8 indicate that comparing SUI with $g_{1}=g_{2}$, the SUI with $g_{2} \gg g_{1}$ is not only able to achieve another $3 \mathrm{~dB}$ improvement, but posses a better loss-tolerance feature.

This loss-insensitive property can be used to split the signal and idler outputs further into more beams for the joint measurement of multiple quantities without a significant reduction of the SNRs. Therefore, the SUI can directly accomplish the quantum enhanced joint measurement for arbitrary number of noncommuting observables. For example, if we further split the signal output into two with a 50/50 BS (see the dashed box in Fig. 6) and place another set of HD (HD3) at the reflection port, we can realize the joint measurement of three noncommuting quadrature-phase amplitudes $X_{m}\left(\theta_{1}\right), X_{m}\left(\theta_{2}\right)$, and $X_{m}\left(\theta_{3}\right)$ with sensitivity beyond SQL using HD1, HD2, and HD3 to simultaneously perform measurement. For example, if we have $g_{1}=1$ and $g_{2}=5$ and each HD device is perfect, the calculated SNRs of measured $X_{m}\left(\theta_{1}\right), X_{m}\left(\theta_{2}\right)$, and $X_{m}\left(\theta_{3}\right)$ surpass the SQL by $7.25 \mathrm{~dB}, 7.6 \mathrm{~dB}$, and $7.25 \mathrm{~dB}$, respectively. The reduction of SNRs in the ports split by the BS is only 0.35 $\mathrm{dB}$ lower than that in the port without splitting.

\section{Postdetection processing for the joint measurement of multiple parameters}

The ability of the SUI scheme to make a measurement of a modulation signal at arbitrary angle $X_{m}(\theta)=X_{m} \cos \theta+$ $Y_{m} \sin \theta$ can be achieved indirectly through the method of postdetection processing as well. The basic principle of the method is to measure a pair of conjugated quadrature amplitudes $X_{m}$ and $Y_{m}$ using HD1 and HD2 at signal and idler output ports. The measurement of $X_{m}(\theta)=X_{m} \cos \theta+Y_{m} \sin \theta$ with a modulation depth $\gamma=X_{m}(\theta)$ is then achieved by processing the photocurrents out of the two sets of HDs. The information $X_{m}(\theta)$ encoded on the probe can be decomposed into phase and amplitude modulations at the same frequency. So the complex amplitude of the probe field is proportional to $1+i \delta-\epsilon$ with $\delta=\gamma \sin \theta$ and $\epsilon=\gamma \cos \theta$. When the two orthogonal quadratures $X_{m}$ and $Y_{m}$ are obtained by measuring $\hat{X}_{s}$ and $\hat{Y}_{i}$ with HD1 and HD2 at the two SUI outputs, we have

$$
\begin{aligned}
\hat{X}_{s} & =\hat{a}_{S_{\text {out }}} e^{-j \varphi_{0}}+\hat{a}_{s_{\text {out }}}^{\dagger} e^{j \varphi_{0}} \\
& =G_{2} \hat{X}_{s_{\text {out } 1}}-g_{2} \hat{X}_{i_{\text {out }}}-G_{2} \hat{X}_{S_{\text {out }}} \epsilon-G_{2} \hat{Y}_{s_{\text {out }}} \delta, \\
\hat{Y}_{i} & =\left(\hat{a}_{i_{\text {out }}} e^{-j \varphi_{0}}-\hat{a}_{i_{\text {out }}}^{\dagger} e^{j \varphi_{0}}\right) / j \\
& =-G_{2} \hat{Y}_{i_{\text {out }}}-g_{2} \hat{Y}_{S_{\text {out }}}-g_{2} \hat{X}_{S_{\text {out }}} \delta+g_{2} \hat{Y}_{S_{\text {out }}} \epsilon,
\end{aligned}
$$

where $e^{j \varphi_{0}} \equiv \alpha /|\alpha|$ is the phase of the seed injection. Using the relation $\hat{X}_{\theta} \equiv \cos \theta \hat{X}_{s}+k \sin \theta \hat{Y}_{i}$, where $k$ is a coefficient that balances the gain difference between the signal and idler ports, we obtain the average signal power and noise fluctuation for the measurement of signal $X_{m}(\theta)$ :

$$
\begin{aligned}
\left\langle\hat{X}_{\theta}\right\rangle^{2} & =\left\langle\left(\cos \theta \hat{X}_{s}+k \sin \theta \hat{Y}_{i}\right)\right\rangle^{2} \\
& =4\left(G_{2} \cos ^{2} \theta+k g_{2} \sin ^{2} \theta\right)^{2} I_{\mathrm{ps}} \gamma^{2}
\end{aligned}
$$

and

$$
\begin{aligned}
\left\langle\Delta \hat{X}_{\theta}^{2}\right\rangle= & {\left[\left(G_{2} G_{1}-g_{1} g_{2}\right)^{2}+\left(G_{1} g_{2}-G_{2} g_{1}\right)^{2}\right] } \\
& \times\left(\cos ^{2} \theta+k^{2} \sin ^{2} \theta\right) .
\end{aligned}
$$

Consequently, we have the SNR

$$
\begin{aligned}
& S N R\left(\hat{X}_{\theta}\right) \\
& =\frac{4\left(G_{2} \cos ^{2} \theta+k g_{2} \sin ^{2} \theta\right)^{2} I_{\mathrm{ps}} \gamma^{2}}{\left[\left(G_{2} G_{1}-g_{1} g_{2}\right)^{2}+\left(G_{1} g_{2}-G_{2} g_{1}\right)^{2}\right]\left(\cos ^{2} \theta+k^{2} \sin ^{2} \theta\right)} .
\end{aligned}
$$

In the case of $k=G_{2} / g_{2}$ and $g_{2} \rightarrow \infty$, Eq. (27) has the simplified form

$$
\operatorname{SNR}\left(\hat{X}_{\theta}\right)=2\left(G_{1}+g_{1}\right)^{2} I_{\mathrm{ps}} \gamma^{2},
$$

which indicates that the quantum enhanced factor obtained by the postdetection is the same as that using direct detection [see Eq. (23)].

The method of postdetection data processing can be extended for the joint measurement of multiple modulation 


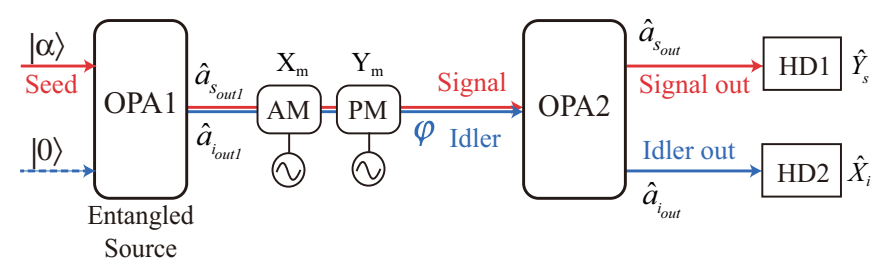

FIG. 9. SUI with dual beams function as the sensing field. OPA, optical parametric amplifier; AM, amplitude modulator; PM, phase modulator; HD, homodyne detection.

signals. For example, if the probe beam carries the modulation information in $N$ different quadrature amplitudes, the measurement of $X_{m}\left(\theta_{1}\right), X_{m}\left(\theta_{2}\right), X_{m}\left(\theta_{3}\right), \ldots$, and $X_{m}\left(\theta_{N}\right)$ can be simultaneously obtained from the calculation of the photocurrent $i(\theta)=i_{1} \cos \theta+k i_{2} \sin \theta$ after substituting $\theta$ with $\theta_{1}, \theta_{2}, \ldots, \theta_{N}$, where $i_{1}$ and $i_{2}$ are the photocurrents out of the HD1 and HD2 when the measurements of $\hat{X}_{s}$ and $\hat{Y}_{i}$ are simultaneously performed. Comparing with the method of directly detecting each quadrature amplitude with a HD, which we have discussed at the end of Sec. III B, the method of postdetection data processing seems more convenience for realizing the joint measurement of noncommuting observables with a number greater than three because there is no need to increase the number of HD devices. However, in practice, the influence of detection efficiency and the noise correlation between the two SUI outputs may introduce extra complexity in the postdetection processing.

\section{SUI with dual-beam sensing}

One unique property of the SUI is that the interference fringe depends on the sum of the phases of the signal and idler beams between two OPAs [18,22]. This suggests that passing both signal and idler fields out of OPA1 through the modulation units of AM and PM will double the signal size at the SUI outputs. This idea is shown in Fig. 9. Different from the SUI in Fig. 6, in which the signal field out of OPA1 functions as the sensing field, the sensing field in Fig. 9 is the two nondegenerate fields produced by OPA1.

In Fig. 9 when the information on phase and amplitude ( $Y_{m}=\delta$ and $X_{m}=\epsilon$ ) encoded on the dual beams out of OPA1 and OPA2 is operated in the deamplification condition, we analyze the SNRs of $Y_{m}$ and $X_{m}$ using HDs at signal and idler outputs to measure, respectively, $\hat{Y}_{s}$ and $\hat{X}_{i}$. We first deduce the intensity of phase signal at the signal output port

$$
\left\langle\hat{Y}_{s}\right\rangle^{2}=4\left(G_{1} G_{2}+g_{1} g_{2}\right)^{2}|\alpha|^{2} \delta^{2} .
$$

Since the noise level at the SUI outputs in Fig. 9 is the same as that in Fig. 6, i.e., $\left\langle\Delta^{2} \hat{Y}_{s}\right\rangle=\left(G_{2} G_{1}-g_{2} g_{1}\right)^{2}+\left(G_{2} g_{1}-\right.$ $\left.g_{2} G_{1}\right)^{2}$, the SNR of $Y_{m}$ measured at the signal output port is

$$
\begin{aligned}
& S N R_{D B}\left(\hat{Y}_{s}\right) \\
& =\frac{4\left(G_{1} G_{2}+g_{1} g_{2}\right)^{2} I_{\mathrm{ps}} \delta^{2}}{\left(G_{1}^{2}+g_{1}^{2}\right)\left[\left(G_{2} G_{1}-g_{2} g_{1}\right)^{2}+\left(G_{2} g_{1}-g_{2} G_{1}\right)^{2}\right]} \\
& \rightarrow 4\left(G_{1}+g_{1}\right)^{2} I_{\mathrm{ps}} \delta^{2} \text { for } g_{1} \gg 1 \text { and } g_{2} \gg g_{1}, \quad
\end{aligned}
$$

where the subscript "DB" refers to the dual-beam scheme, and the photon number of sensing field $I_{\mathrm{ps}}=\left(G_{1}^{2}+\right.$ $\left.g_{1}^{2}\right)|\alpha|^{2}\left(|\alpha|^{2} \gg 1\right)$ is the total intensity of the signal and idler fields out of OPA1. Moreover, under the same condition, it is straightforward to calculate the intensity of the amplitude signal at the idler output port

$$
\left\langle\hat{X}_{i}\right\rangle^{2}=4\left(G_{1} g_{2}-g_{1} G_{2}\right)^{2} I_{\mathrm{ps}} \epsilon^{2},
$$

which shows that the intensity of amplitude modulation at the SUI idler output is negligibly small. Particularly, for the case of $g_{1} \gg 1, g_{2} \gg 1$, we have $\left\langle\hat{X}_{i}\right\rangle^{2} \rightarrow 0$. This is caused by the common mode rejection in intensity fluctuation when OPA2 in Fig. 9 is operated at a deamplification condition. Indeed, each SUI output field in Fig. 9 carries the same information. If we measure $\hat{Y}_{i}$ in the idler output, the same SNR as measuring $\hat{Y}_{s}$ for the phase modulation can be obtained. So $\hat{Y}_{i}$ can be viewed as an exact copy of $\hat{Y}_{s}$, which means the phase information carried by the sensing field can be split into two without adding noise [22].

The above results indicate that the dual-beam scheme in Fig. 9 cannot realize the quantum enhanced joint measurement of phase and amplitude modulations. However, for the phase measurement only, the improvement factor over SQL is twice that of SUI in Fig. 6.

\section{E. A rule of resource conservation for joint measurement}

Comparing the measurement realized using the quantum schemes in Figs. 6 and 9, we find the relation $S N R_{S U I}\left(\hat{X}_{s}\right)+$ $S N R_{S U I}\left(\hat{Y}_{i}\right)=S N R_{D B}\left(\hat{Y}_{S}\right)$ holds for the case of equal modulation strength $\delta=\epsilon$, which means that the total sum of the SNRs for joint measurement equals the SNR for one observable obtained by consuming all the resource on it. In fact, the comparison of the classical measurement schemes in Figs. 1-3 also reveals this resource partition relation, as shown in Eqs. (3) and (10).

To better understand the resource conservation rule discussed above, let us consider if the rule applies for the quantum resource consumed in the dense coding scheme in Fig. 5. Hence, we try to figure out a scheme in which the entanglement generated by an OPA is fully used to measure one observablephase or amplitude-as precisely as possible. As shown in Fig. 10, the entangled signal and idler fields copropagate and function as the probe for carrying information. After sending the dual beam, encoded with the phase and amplitude signals ( $Y_{m}=\delta$ and $X_{m}=\epsilon$ ) by weakly modulating PM and AM, through a dual-band filter F2, signal and idler fields are then separated and detected, respectively, by HD1 and HD2. The quadrature amplitudes $\hat{X}_{s, i}$ and $\hat{Y}_{s, i}$ of signal and idler fields are

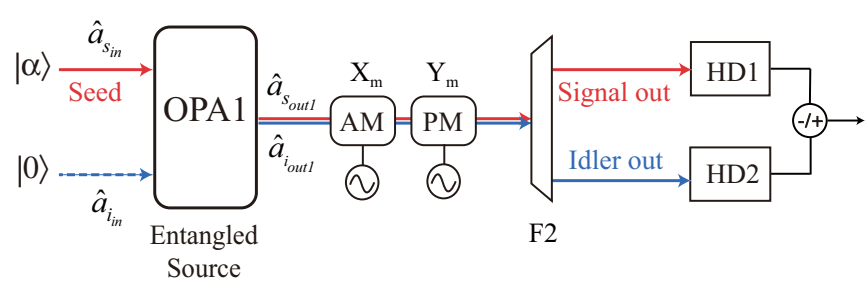

FIG. 10. Measurement scheme of using entanglement to measure one observable as precisely as possible. AM, amplitude modulator; PM, phase modulator; OPA, optical parametric amplifier; F2, dualband filter; HD, homodyne detection. 
measured by setting the two HDs at $\phi_{1,2}=0$ and $\phi_{1,2}=\pi / 2$, respectively. To reduce the noise level in the measurement, we combine the photocurrents of two HDs with a subtractor or adder so that the quantum noise cancellation due to quantum correlation via $\hat{X}_{s}-\hat{X}_{i}$ and $\hat{Y}_{s}+\hat{Y}_{i}$ can be utilized.

When the photocurrents of HD1 and HD2 with LO phase locked at $\phi_{1,2}=\pi / 2$ are added, we have the power of the phase signal $Y_{m}$ :

$$
\left\langle\hat{Y}_{s}+\hat{Y}_{i}\right\rangle^{2}=4(G+g)^{2}|\alpha|^{2} \delta^{2} .
$$

The corresponding noise is

$$
\left\langle\Delta\left(\hat{Y}_{s}+\hat{Y}_{i}\right)\right\rangle^{2}=2(G-g)^{2}=\frac{2}{(G+g)^{2}} .
$$

From the Eqs. (32) and (33), it is straightforward to obtain the SNR for the measured phase modulation

$$
\begin{aligned}
S N R_{D B-D C}(\hat{Y}) & =\frac{2(G+g)^{4} I_{\mathrm{ps}} \delta^{2}}{G^{2}+g^{2}} \\
& \rightarrow 4(G+g)^{2} I_{\mathrm{ps}} \delta^{2} \text { for } g \gg 1,
\end{aligned}
$$

where the subscript "DB-DC" represents the scheme in Fig. 10. On the other hand, when the photocurrents of HD1 and HD2 with LO phase locked at $\phi_{1,2}=0$ are subtracted, the measurement noise $\left\langle\Delta\left(\hat{X}_{s}-\hat{X}_{i}\right)\right\rangle^{2}=\frac{2}{(G+g)^{2}}$ is lower than the SNL. However, in this case, the power of amplitude signal $X_{m}$, which can be expressed as $\left\langle\hat{X}_{s}-\hat{X}_{i}\right\rangle^{2}=4(G-g)^{2}|\alpha|^{2} \epsilon^{2}$, is negligibly small, particularly in the high-gain regime. Therefore, the measurement results are the same as those in Eqs. (30) and (31).

Notice that the scheme in Fig. 10 can be used to measure only the phase with sensitivity beyond SQL. Comparing with the dense coding scheme for joint measurement in Fig. 5, we find the relation $S N R_{D C}\left(\hat{X}_{b_{1}}\right)+S N R_{D C}\left(\hat{Y}_{b_{2}}\right)=$ $S N R_{D B-D C}(\hat{Y})$ holds for the case of $\delta=\epsilon$, which again demonstrates the joint measurement can be viewed as the partition of total resources, i.e., the fixed amount of noise reduction factor of $1 /(G+g)^{2}$ originated from the EPRcorrelated source (OPA1) is distributed among the measurement of two jointly measured quantities.

\section{HEISENBERG LIMIT FOR THE JOINT MEASUREMENT OF PHASE AND AMPLITUDE}

It has been known from the very beginning that the sensitivity of the SUI in phase measurement is bounded by the Heisenberg limit when there is no intra-interferometer loss and no seed injection $[15,17,24]$. Now we will study the Heisenberg limit for the joint measurement realized using the SUI in Fig. 6.

In order to reach the Heisenberg limit in the joint measurement, similar to the phase measurement [15,24], the OPA 1 of the SUI must be operated without input, i.e., the intensity of the seed input field in Fig. 6 is $|\alpha|^{2}=0$. In this case, the photon number of the probe signal beam is $N=I_{\mathrm{ps}}=g_{1}^{2}$, and the measured powers of amplitude and phase signals in Eq. (18) are rewritten as

$$
\left\langle\hat{X}_{S_{\text {out }}}\right\rangle^{2}=4 G_{2}^{2} g_{1}^{2} \epsilon^{2},\left\langle\hat{Y}_{i_{\text {out }}}\right\rangle^{2}=4 g_{2}^{2} g_{1}^{2} \delta^{2} .
$$

In this case, for the SUI operating at the deamplification condition with $g_{2} \rightarrow \infty$, the SNRs of measured phase and amplitude in Eq. (20) have a simplified form:

$$
\begin{aligned}
& S N R_{\delta}=\frac{\left\langle\hat{X}_{S_{\text {out }}}\right\rangle^{2}}{\left\langle\Delta^{2} \hat{X}_{S_{\text {out }}}^{2}\right\rangle}=2\left(G_{1}+g_{1}\right)^{2} g_{1}^{2} \delta^{2}, \\
& S N R_{\epsilon}=\frac{\left\langle\hat{Y}_{\text {out }}\right\rangle^{2}}{\left\langle\Delta^{2} \hat{Y}_{i_{\text {out }}}^{2}\right\rangle}=2\left(G_{1}+g_{1}\right)^{2} g_{1}^{2} \epsilon^{2} .
\end{aligned}
$$

Under the condition of $S N R_{\delta}=S N R_{\epsilon}=1$ and $g_{1} \gg 1$, we obtain the minimum detectable amplitude and phase signals encoded on the probe signal beam, $\epsilon_{m}=1 / 2 \sqrt{2} N$ and $\delta_{m}=$ $1 / 2 \sqrt{2} N$, which are the Heisenberg limit of the joint measurement.

On the other hand, when the losses inside the interferometer (transmission losses between two OPAs) are included, the performance of SUI will be severely affected. For simplicity, assuming the losses of the two arms in SUI, labeled signal and idler, are equal, we then model the loss $L$ as a BS with transmissivity of $T=1-L$. After some algebra, it is straightforward to deduce powers of the amplitude and phase modulation measured at the signal and idler outputs:

$$
\left\langle\hat{X}_{s}\right\rangle^{2}=4(1-L) G_{2}^{2} g_{1}^{2} \epsilon^{2}, \quad\left\langle\hat{Y}_{i}\right\rangle^{2}=4(1-L) g_{2}^{2} g_{1}^{2} \delta^{2},
$$

and the corresponding noise fluctuations are given by

$$
\begin{aligned}
\left\langle\Delta^{2} \hat{X}_{s}\right\rangle= & \left\langle\Delta^{2} \hat{Y}_{i}\right\rangle \\
= & (1-L)\left[\left(G_{2} G_{1}-g_{1} g_{2}\right)^{2}+\left(G_{1} g_{2}-G_{2} g_{1}\right)^{2}\right] \\
& +L\left(G_{2}^{2}+g_{2}^{2}\right) .
\end{aligned}
$$

Under the condition of $g_{2} \rightarrow \infty$, the SNRs of measured amplitude and phase are then expressed as

$$
\begin{aligned}
S N R_{S U I-\text { Loss }}\left(\hat{X}_{s}\right) & =\frac{2(1-L) I_{\mathrm{ps}} \epsilon^{2}}{(1-L)\left(G_{1}-g_{1}\right)^{2}+L}, \\
S N R_{S U I-\operatorname{Loss}}\left(\hat{Y}_{i}\right) & =\frac{2(1-L) I_{\mathrm{ps}} \delta^{2}}{(1-L)\left(G_{1}-g_{1}\right)^{2}+L},
\end{aligned}
$$

where $I_{\mathrm{ps}}=g_{1}^{2}$. Setting the values of SNRs to 1 , we find the minimum measurable modulation of amplitude and phase signals are

$$
\begin{aligned}
\epsilon_{m}=\delta_{m} & =\sqrt{\frac{1}{2 I_{\mathrm{ps}}\left(G_{1}+g_{1}\right)^{2}}+\frac{L}{2(1-L) I_{\mathrm{ps}}}} \\
& =\sqrt{\frac{1}{2 I_{\mathrm{ps}}^{2}(1+\lambda)^{2}}+\frac{L}{2(1-L) I_{\mathrm{ps}}}}
\end{aligned}
$$

with $\lambda \equiv G_{1} / g_{1}=\sqrt{\left(I_{\mathrm{ps}}+1\right) / I_{\mathrm{ps}}}$. To demonstrate the influence of loss $L$, we plot the minimum measurable signal, $\epsilon_{m}$ or $\delta_{m}$, as a function of $I_{\mathrm{ps}}$ when the value of $L$ is different, as shown in Fig. 11. For the convenience of comparison, we also depict the SQL $\left(1 / \sqrt{2 I_{\mathrm{ps}}}\right)$ and HL [achieved by substituting $L=0$ in Eq. (40)] in Fig. 11. It is obvious that for both the classical limit of SQL and quantum limit of HL, the sensitivity, represented by the minimal measurable $\epsilon_{m}$ and $\delta_{m}$, increases with the photon number of probe field $I_{\mathrm{ps}}$. When the intensities of sensing fields are the same, the ratio of the sensitivity for $\mathrm{HL}$ and SQL increases with $I_{\mathrm{ps}}$. In other words, the quantum enhancement factor increases with $I_{\mathrm{ps}}$. Moreover, we find that for the SUI with internal loss $L$, the Heisenberg limit of $\sim 1 / 2 \sqrt{2} I_{\mathrm{ps}}$ is achievable only when $I_{\mathrm{ps}}<1 / L$, whereas the 


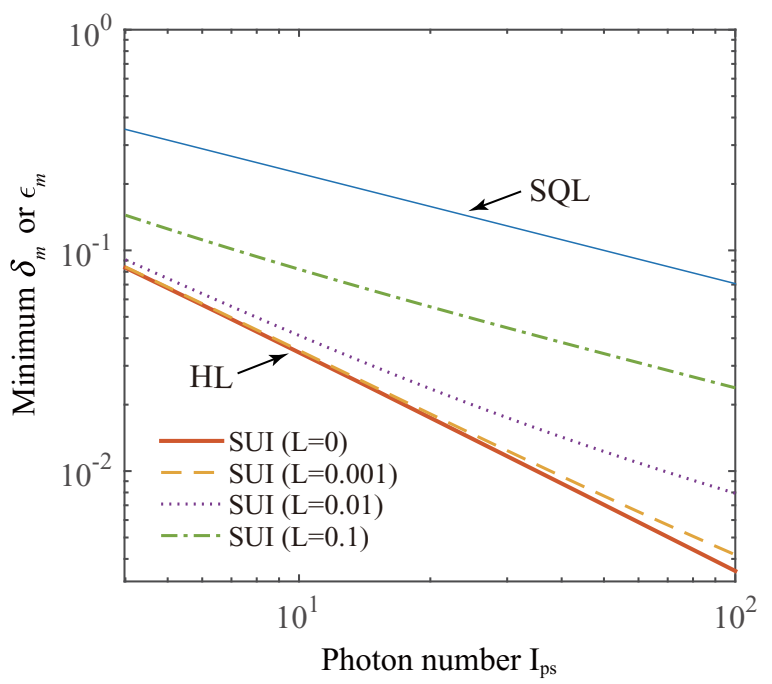

FIG. 11. Minimum measurable amplitude and phase signals, $\epsilon_{m}$ and $\delta_{m}$, as a function of the photon number of the probe field $I_{\mathrm{ps}}$ for the SUI with different internal losses $L$. SQL, standard quantum limit; HL, Heisenberg limit.

SQL of $\sim 1 / \sqrt{2 I_{\mathrm{ps}}}$ will be approached when $I_{\mathrm{ps}} \gg 1 / L$. The results indicate that if the photon number of sensing field $I_{\mathrm{ps}}$ is low, the deduction of the quantum enhanced factor is not significant. However, if the photon number of $I_{\mathrm{ps}}$ is high, the quantum enhancement factor will be dramatically affected by the loss $L$. For example, when the photon number of $I_{\mathrm{ps}}$ is 4 , a $10 \%$ loss inside the SUI increases $\epsilon_{m}$ and $\delta_{m}$ only by about 1.7 times that of HL. On the other hand, when the photon number of $I_{\mathrm{ps}}$ is $100, a 10 \%$ loss will increase $\epsilon_{m}$ and $\delta_{m}$ by about 6.8 times that of $\mathrm{HL}$, although the increase of $I_{\mathrm{ps}}$ from four to 100 photons increases the sensitivity by about six times. Therefore, for the practical application, the SUI with a stronger sensing field is preferred, but its sensitivity for joint measurement is limited by the losses inside the interferometer. This is consistent with previous studies $[24,26]$.

\section{CONCLUSION}

In summary, we have investigated various schemes for joint measurement of multiple noncommuting observables with both classical sources and quantum sources and compared their performance under the condition of the same probe intensity. We find that quantum schemes using entangled sources have significant improvement in the SNR over the classical schemes. The dense coding scheme with frequency degenerated entanglement as the quantum source is vulnerable to losses. However, the developed SUI having the ability to mix coherently two entangled fields with different wavelengths is insensitive to the losses outside the interferometer such as propagation and detection losses. Moreover, the SUI can be extended for joint measurement of multiple $(>2)$ quadraturephase amplitudes with arbitrary angles. Furthermore, in our investigation, we find an interesting effect of resource partition in the joint measurement of two orthogonal observables; that is, the sum of the SNRs for joint measurement of two orthogonal observables is equal to the SNR of one observable measured by consuming all the measurement resource on it. This rule applies to both classical and quantum schemes.

\section{ACKNOWLEDGMENTS}

The work is supported in part by the National Key Research and Development Program of China (2016YFA0301403), the 973 program of China (2014CB340103), the National Natural Science Foundation of China $(91736105,11527808)$, and the 111 project (B07014).
[1] C. M. Caves, Quantum-mechanical noise in an interferometer, Phys. Rev. D 23, 1693 (1981).

[2] C. K. Hong, S. R. Friberg, and L. Mandel, Optical communication channel based on coincident photon pairs, Appl. Opt. 24, 3877 (1985).

[3] M. Xiao, L.-A. Wu, and H. J. Kimble, Precision Measurement Beyond the Shot-Noise Limit, Phys. Rev. Lett. 59, 278 (1987).

[4] P. Grangier, R. E. Slusher, B. Yurke, and A. LaPorta, SqueezedLight-Enhanced Polarization Interferometer, Phys. Rev. Lett. 59, 2153 (1987).

[5] K. Goda, O. Miyakawa, E. E. Mikhailov, S. Saraf, R. Adhikari, K. McKenzie, R. Ward, S. Vass, A. J. Weinstein, and N. Mavalvala, A quantum-enhanced prototype gravitational-wave detector, Nat. Phys. 4, 472 (2008).

[6] A. Einstein, B. Podolsky, and N. Rosen, Can quantummechanical description of physical reality be considered complete? Phys. Rev. 47, 777 (1935).

[7] M. D. Reid, Demonstration of the Einstein-Podolsky-Rosen paradox using nondegenerate parametric amplification, Phys. Rev. A 40, 913 (1989).

[8] Z. Y. Ou, S. F. Pereira, H. J. Kimble, and K. C. Peng, Realization of the Einstein-Podolsky-Rosen Paradox for Continuous Variables, Phys. Rev. Lett. 68, 3663 (1992).
[9] S. L. Braunstein and H. J. Kimble, Dense coding for continuous variables, Phys. Rev. A 61, 042302 (2000).

[10] J. Zhang and K. Peng, Quantum teleportation and dense coding by means of bright amplitude-squeezed light and direct measurement of a Bell state, Phys. Rev. A 62, 064302 (2000).

[11] X. Li, Q. Pan, J. Jing, J. Zhang, C. Xie, and K. Peng, Quantum Dense Coding Exploiting a Bright Einstein-Podolsky-Rosen Beam, Phys. Rev. Lett. 88, 047904 (2002).

[12] S. Steinlechner, J. Bauchrowitz, M. Meinders, H. MüllerEbhardt, K. Danzmann, and R. Schnabel, Quantum-dense metrology, Nat. Photon. 7, 626 (2013).

[13] Z. Y. Ou, Quantum amplification with correlated quantum fields, Phys. Rev. A 48, R1761 (1993).

[14] J. Kong, F. Hudelist, Z. Y. Ou, and W. Zhang, Cancellation of Internal Quantum Noise of an Amplifier by Quantum correlation, Phys. Rev. Lett. 111, 033608 (2013).

[15] B. Yurke, S. L. McCall, and J. R. Klauder, SU(2) and SU(1,1) interferometers, Phys. Rev. A 33, 4033 (1986).

[16] Z. Y. Ou, Fundamental quantum limit in precision phase measurement, Phys. Rev. A 55, 2598 (1997).

[17] B. E. Anderson, B. L. Schmittberger, P. Gupta, K. M. Jones, and P. D. Lett, Optimal phase measurements with bright- 
and vacuum-seeded SU(1,1) interferometers, Phys. Rev. A 95, 063843 (2017).

[18] F. Hudlist, J. Kong, C. Liu, J. Jing, Z. Y. Ou, and W. Zhang, Quantum metrology with parametric amplifier-based photon correlation interferometers, Nat. Commun. 5, 3049 (2014).

[19] A. M. Marino, N. V. Corzo Trejo, and P. D. Lett, Effect of losses on the performance of an SU(1,1) interferometer, Phys. Rev. A 86, 023844 (2012).

[20] B. E. Anderson, P. Gupta, B. L. Schmittberger, T. Horrom, C. Hermann-Avigliano, K. M. Jones, and P. D. Lett, Phase sensing beyond the standard quantum limit with a variation on the SU(1,1) interferometer, Optica 4, 752 (2017).

[21] W. N. Plick, J. P. Dowling, and G. S. Agarwal, Coherent-lightboosted, sub-shot noise, quantum interferometry, New J. Phys. 12, 083014 (2010).
[22] X. Guo, X. Li, N. Liu, and Z. Y. Ou, Quantum information tapping using a fiber optical parametric amplifier with noise figure improved by correlated inputs, Sci. Rep. 6, 30214 (2016).

[23] C. M. Caves, Quantum limits on noise in linear amplifiers, Phys. Rev. D 26, 1817 (1982).

[24] Z. Y. Ou, Enhancement of the phase-measurement sensitivity beyond the standard quantum limit by a nonlinear interferometer, Phys. Rev. A 85, 023815 (2012).

[25] M. Manceau, G. Leuchs, F. Khalili, and M. Chekhova, Detection loss tolerant supersensitive phase measurement with an SU(1,1) interferometer, Phys. Rev. Lett. 119, 223604 (2017).

[26] R. Demkowicz-Dobrzanski, J. Kolodynski, and M. Guta, The elusive Heisenberg limit in quantum enhanced metrology, Nat. Commun. 3, 1063 (2012). 